\title{
Antimicrobial resistance in Haemophilus influenzae
}

\author{
MAIR POWELL
}

Department of Medical Microbiology, The London Hospital Medical College, Turner Street, London E1 2AD

Since the early 1970 s, recognition of the increasing prevalence of resistance to several antimicrobial agents among clinical isolates of Haemophilus influenzae in many countries has brought about changes in prescribing practices. Direct comparison of susceptibility test results from various centres cannot be made without consideration of the factors known to have a crucial effect on the apparent invitro resistance of this species to many antimicrobial agents. These include the medium, antibiotic concentration in the disk and, particularly, the size of inoculum used. The variety of zone diameters and minimum inhibitory concentrations (MICs) employed as susceptibility breakpoints in different studies reflects not only the range of methods used but also the lack of agreement on the relationship of in-vitro results to in-vivo efficacy of an antimicrobial agent against $H$. influenzae.

Despite the problems of interpretation of results, it has become clear that isolates of $H$. influenzae of Pittman (capsule) type b merit separate consideration because of their propensity to cause invasive infections. Several national studies have reported a consistently higher prevalence of $\beta$-lactamase production among isolates of type $b$ when compared with non-capsulate $H$. influenzae (Doern et al., 1986; Powell et al., 1987; Machka et al., 1988) and some have found that type-b isolates are more likely to be resistant to other antimicrobial agents (Dabernat et al., 1986). Although they are responsible for most cases of invasive disease, this difference is also seen between type-b isolates producing non-invasive disease and the non-capsulate organisms more commonly responsible for less serious infections. Hence, differentiation of type-b isolates from those which are non-capsulate or of other capsule types is an important consideration in any study of the prevalence and nature of antimicrobial resistance in this species and is crucial in the interpretation of the prevalence data.

Received 18 Feb. 1988; accepted 29 Mar. 1988.

\section{Resistance to $\beta$-lactams}

$H$. influenzae type-b infections which failed to respond to ampicillin therapy prompted some of the earliest reports of resistance to antimicrobial agents in use against this species (Gunn et al., 1974). The prevalence of $\beta$-lactamase-producing $H$. influenzae identified from United Kingdom surveys rose from $1.6 \%$ in 1977 (Howard et al., 1978) to $18 \%$ amongst type-b isolates and 6.2\% overall in 1986 (Powell et al., 1987). In Europe, $\beta$-lactamase production is most common among type-b $(64 \%)$ and non-capsulate $(26 \%)$ Spanish isolates (Machka et al., 1988). Spain has a particular problem in the Barcelona area where $57 \%$ of type-b isolates responsible for meningitis are $\beta$-lactamase positive and also resistant to chloramphenicol (Campos et al., 1986). A multi-centre study in the USA reported that, overall, $32 \%$ of type-b and $16 \%$ of noncapsulate clinical isolates were $\beta$-lactamase positive (Doern et al., 1988) but noted a geographical variation in prevalence among type-b isolates from $<10 \%$ to $>90 \%$.

Medeiros and O'Brien (1975) established that the enzymes present in some early $\beta$-lactamase producers were TEM-like in character. De Graaff et al. (1976) found that the transposon coding for $\beta$ lactamase production could be located on either large or small plasmids of mol. wts $\left(10^{6}\right) 30$ and 3 respectively, which were otherwise unrelated in their nucleotide sequences. Several workers, however, have demonstrated that the genes encoding $\beta$ lactamase and resistance to several other antimicrobial agents in $H$. influenzae are not always situated on extrachromosomal DNA. Stuy (1980) found that isolates with several different resistance patterns were frequently free of detectable plasmids. Murphey-Corb et al. (1984) failed to detect extrachromosomal DNA in 43 of $66 \beta$-lactamase-producing isolates of $H$. influenzae. The plasmid-free isolates conjugally transferred resistance to an ampicillinsusceptible recipient at a lower frequency than their plasmid-containing counterparts but offspring of 
both types of donor contained extrachromosomal DNA as single bands (mol. wt $30 \times 10^{6}$ ). By restriction-enzyme analysis and Southern-hybridisation techniques, entire plasmid sequences were detected within the chromosome of four plasmidfree $\beta$-lactamase-producing type-b isolates. Mendelman et al. (1985) also found that whereas most (29 of 36) Alaskan $\beta$-lactamase-producing $H$. influenzae isolates of type $\mathrm{b}$ were plasmid-free, many of the resistant transconjugants obtained harboured extrachromosomal DNA.

The demonstration by Roberts and Smith (1980) that large plasmids in $H$. influenzae are easily overlooked if a single method of lysis is used raised worries that plasmid DNA sometimes co-migrated with chromosomal DNA giving an alternative explanation for probe hybridisations with chromosomal bands. However, Mendelman et al. (1985) were able to show in their study, and during an investigation of ampicillin- and chloramphenicolresistant isolates (Mendelman et al., 1984a), that plasmid probes bearing specific R-determinants hybridised with both the chromosomal and extrachromosomal DNA of those isolates of $H$. influenzae found to contain plasmids.

Whilst the mechanism of cell-to-cell transfer of chromosomal gene sequences coding for $\beta$-lactamase production and resistance to other antimicrobial agents has not been fully elucidated, transformation of $H$. influenzae by haemophilus DNA has been shown to be an efficient process involving uptake of transforming DNA into cellsurface membranous structures (transformasomes) which afford some degree of protection against enzymic degradation (Barany and Kahn, 1985). Thus, a hypothesis that implicates transformation in cell-to-cell transfer of chromosomal gene sequences is attractive but it is not known to what extent this process occurs in vivo.

Production of a TEM- $1 \quad \beta$-lactamase is the predominant, but not the sole, mechanism of ampicillin resistance in $H$. influenzae. In 1981, Rubin et al. reported the presence of a novel $\beta$ lactamase, later called $R O B-1$, in a type-b isolate causing meningitis. The enzyme was not detected by the initial cell-suspension chromogenic-cephalosporin assay but did produce a positive result when the test was repeated with a very heavy inoculum. Iso-electric focussing demonstrated that ROB-1 had a very different pI $(8 \cdot 1)$ from that of TEM-1 (5.4) and slightly different $\beta$-lactamhydrolysis rates. Production of the novel enzyme was lost after curing a similar-sized plasmid identified in two isolates from the patient's blood and cerebrospinal fluid. A possible source for ROB-
1 was suggested when Medeiros et al. (1986) discovered that ampicillin-resistant $H$. pleuropneumoniae, a swine pathogen, also produced ROB-1 and that the plasmids encoding the enzyme in both Haemophilus species were closely homologous.

Early reports of resistance to ampicillin that was not mediated by $\beta$-lactamase production included that found in several non-capsulate isolates from respiratory specimens (Bell and Plowman, 1980 ) and in sporadically occurring type-b isolates (Markowitz, 1980; Offit et al., 1982). In a survey in the UK in 1986 (Powell et al., 1987) 100 isolates $(4.1 \%)$ were identified that showed reduced zone diameters $(<20 \mathrm{~mm})$ to $2-\mu \mathrm{g}$ ampicillin disks and required ampicillin concentrations between 1 and $64 \mathrm{mg} / \mathrm{L}$ for inhibition. These isolates appear to be generally uncommon in Europe (Machka et al., 1988) and the USA (Doern et al., 1988), but it is not possible to gauge accurately the worldwide prevalence of this non-lactamase-mediated ("intrinsic") resistance because of the problems which have become apparent in its detection. Establishment of a resistance breakpoint for these isolates must be referenced to susceptibility-testing methods adopted and is complicated by the observation that they rarely require more ampicillin than $4 \mathrm{mg} / \mathrm{L}$ for inhibition (Powell and Williams, 1988).

Attempts to identify the mechanism responsible for this type of resistance have concentrated on differences in penicillin-binding and outer-membrane proteins (PBPs and OMPs) between these isolates and those susceptible to ampicillin. PBP changes noted in a type-b isolate studied by Parr and Bryan (1984) and in transformants derived from four non-capsulate isolates investigated by Mendelman et al. (1984b) suggested that such differences played an important role in nonlactamase-mediated resistance. A chromosomal location for the genes determining resistance was postulated. It is unfortunate that interpretation of PBP studies is complicated by the natural variation seen in $H$. influenzae. Whilst both Makover et al. (1981) and Serfass et al. (1986) identified eight major PBPs in this species, the latter study demonstrated a considerable variation in profiles among ampicillin-susceptible isolates. These differences were even more pronounced among those with non-lactamase-mediated resistance although a strong association with a change in PBP 5 was noted.

Outer-membrane protein profiles of both type-b and non-capsulate isolates have proved to be even more diverse (Loeb and Smith, 1980). Although Reid et al. (1987) have recently documented profile differences between ampicillin-susceptible and am- 
Table I. Modal MICs of $\beta$-lactamase-stable antimicrobial agents against $H$. influenzae

\begin{tabular}{ll}
\hline Antimicrobial agent & $\begin{array}{c}\text { Modal MIC for 2458 isolates } \\
(\mathrm{mg} / \mathrm{L})\end{array}$ \\
\hline $\begin{array}{l}\text { Amoxycillin and clavulanic } \\
\text { acid }(2: 1)\end{array}$ & $0 \cdot 5$ \\
Cefaclor & $4 \cdot 0$ \\
Cefixime & $0 \cdot 03$ \\
Aztreonam & 0.06 \\
Imipenem & $1 \cdot 0$ \\
\hline
\end{tabular}

picillin-resistant, $\beta$-lactamase-negative isolates of the same biotype, no specific protein change has been implicated as being responsible for this phenomenon. Indeed, the variable reduction in susceptibility of isolates of this type (Powell and Williams, $1987 a, b$ ) (see tables I and II) to other $\beta$ lactams supports the conclusion, inferred by the investigations described, that several different mechanisms may be involved. In addition, the degree of genetic and phenotypic diversity among $\beta$-lactamase-negative, ampicillin-resistant, nontypable $H$. influenzae, based on results from multilocus enzyme electrophoresis, auxotroph testing and PBP profiles, has excluded the possibility of a single recent origin for these isolates (Mendelman et al., 1987).

Since the advent of ampicillin resistance in $H$. influenzae, many $\beta$-lactamase-stable compounds have proved to be highly active in vitro and in vivo against ampicillin-susceptible and resistant $\beta$-lactamase-positive organisms. The implications of the finding that ampicillin-resistant, $\beta$-lactamase-negative isolates show reduced susceptibility to many of these compounds have yet to be evaluated. The reduction in susceptibility observed is rarely of such a degree that these isolates can be considered resistant on in-vitro testing according to the criteria established for any one agent (Powell and Williams, 1988). Whether differences between the modal MICs of various $\beta$-lactams among these isolates and the corresponding modal values for the ampicillin-susceptible population are sufficient to give rise to treatment failures will be most crucial when these agents are used for treatment of infections at sites where their penetration is unreliable or poor.

\section{Chloramphenicol}

The rising prevalence of ampicillin resistance, particularly among type-b isolates, promoted the use of chloramphenicol as first-line therapy of invasive $H$. influenzae disease. Although resistance to chloramphenicol has been reported quite frequently since the mid-1970s in both type-b and noncapsulate isolates, its prevalence is still low in many countries. Doern et al. (1986) reported resistance in $<1 \%$ of isolates in the USA, Powell et al. (1987) in $<2 \%$ of isolates in the UK and Machka et al. (1988) in $<2 \%$ of isolates in most European countries with the exception of Belgium ( $11 \%$ in type-b and non- $b$ isolates) and Spain (41\% in type b, $22 \%$ in non-b isolates).

Early investigations established that resistance to chloramphenicol was almost invariably accom-

Table II. Reduction in susceptibility to $\beta$-lactamase-stable agents and relationship to susceptibility to ampicillin in $H$. influenzae

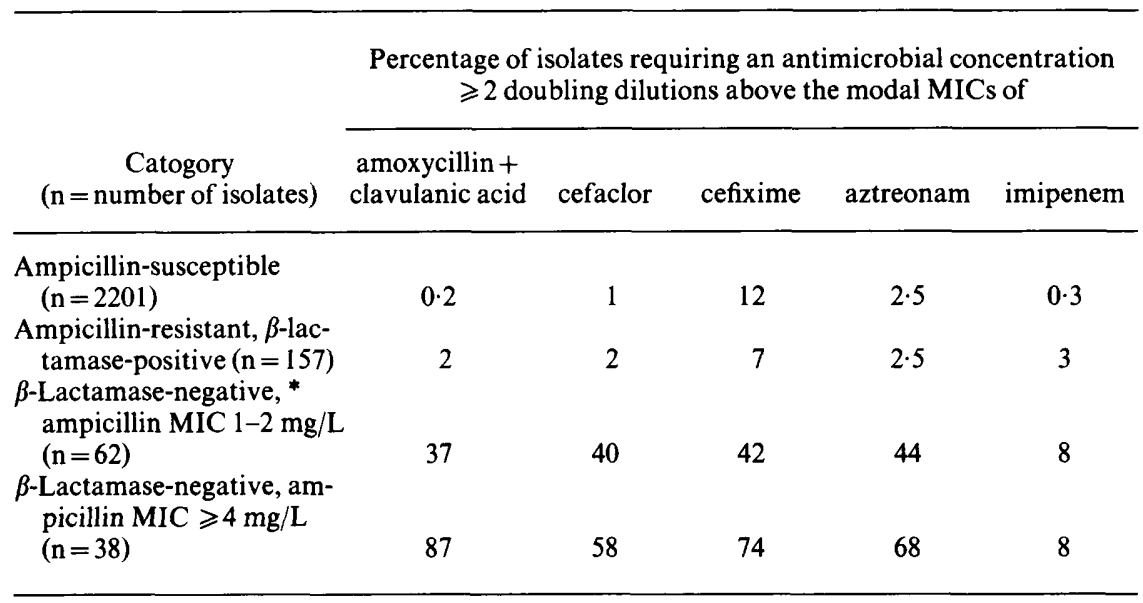

*Reduced susceptibility to ampicillin as indicated by zone diameter $<20 \mathrm{~mm}$ to $2 \mu \mathrm{g}$ ampicillincontaining disk. 
panied by tetracycline resistance and both $\mathrm{R}$ determinants were transferred by large plasmids of mol. wt $\left(10^{6}\right)$ 34-46 (van Klingeren et al., 1977; Roberts et al., 1980). Roberts et al. (1980, 1982) determined that all chloramphenicol acetyltransferases (CATs) mediating resistance in $H$. influenzae collected from many countries resembled the typeII CATs of enterobacteria in their sensitivity to inhibition by 5,5'-dithiobis-2-nitrobenzoate (DTNB) but not all CAT-producing strains of $H$. influenzae possess detectable extrachromosomal DNA. The work of Mendelman et al. (1984a) already described provided supportive evidence for frequent chromosomal integration of the chloramphenicol R-determinant. Examination of 42 chloramphenicol-resistant isolates collected during a survey in the UK in 1986 established that, although all of them produced a type II-like CAT constitutively, only five had visually-detectable extrachromosomal DNA as single plasmids (mol. wt $40 \times$ $10^{6}$ ), despite the application of three different methods of plasmid extraction to eight isolates (Powell and Livermore, 1988). Moreover, ethidium bromide-curing of plasmids in two isolates originally found to contain extrachromosomal DNA did not affect their original resistance patterns, suggesting that chromosomal integration of the CAT genes is a common phenomenon among UK isolates.

In 1985, Burns et al. described four non-capsulate isolates of $H$. influenzae from blood and sputum that did not produce CAT but required chloramphenicol concentrations of $\geqslant 20 \mathrm{mg} / \mathrm{L}$ for inhibition. A decreased rate of chloramphenicol uptake by resistant isolates was demonstrated and, after outer membrane-protein analyses, it was suggested that marked reduction in one protein (mol. wt $40 \times$ $10^{3}$ ), shown to function as a porin by Vachon et al. (1985), might correlate with chloramphenicol resistance mediated by decreased permeability. Isolates of this type have not been reported from the UK and their prevalence is unknown.

\section{Tetracycline}

Tetracycline resistance remains at $<3 \%$ in the UK (Powell et al., 1987). Although resistance to tetracycline may occur without resistance to chloramphenicol, a high prevalence is generally seen only in those areas where the latter is high. Jahn et al. (1979) found that production and carriage of more than one copy on a plasmid of the transposon encoding resistance to tetracycline followed exposure to this antibiotic and correlated with a higher MIC for both tetracycline and chloramphenicol. The results of hybridisation studies indicated that the transposon determining tetracycline resistance served as a carrier for the CAT gene and in some isolates the transposon for $\beta$-lactamase production had become integrated in a similar fashion.

Marshall et al. (1984) reported that 35 of 40 isolates of $H$. influenzae examined showed transferable plasmid-mediated resistance to tetracycline. Hybridisation occurred between a class-B tetracycline-resistance determinant probe, generally found on transposon Tn10, and all 35 isolates which could transfer their resistance as well as two which possessed a non-transferable $\mathrm{R}$-determinant. In contrast with the inducible resistance found in other species carrying Tn10, these isolates of $H$. influenzae showed only constitutive expression of resistance. The same phenomenon has also been observed in $H$. parainfluenzae carrying Tn10 (Levy et al., 1984) and in which it was ascribed to lack of an active repressor molecule.

The remaining three of the 40 isolates examined possessed non-transferable tetracycline resistance and failed to hybridise with any of the four probes derived from other gram-negative organisms. Two of these were also unusual in showing amplification of resistance after incubation with increasing concentrations of tetracycline. Hence, several mechanisms may mediate resistance to tetracyline in this species and studies already described have suggested that there may be frequent integration of the R-determinant in the chromosome.

\section{Anti-folate agents}

The prevalence of resistance to trimethoprim in the UK reached $4.2 \%$ in 1986 (Powell et al., 1987). Few international studies have reported on resistance to trimethoprim not tested in combination with sulphamethoxazole. Campos and GarciaTornel (1987) found that $55 \%$ of 83 capsulate and non-capsulate isolates from Spanish children were resistant to five antimicrobial agents including trimethoprim, indicating the presence of a major problem. Prevalence figures, however, for resistance to trimethoprim in combination with sulphamethoxazole from other countries suggest that the Spanish experience is most unusual (Doern et al., 1988).

Kirven and Thornsberry (1978), using a microdilution-broth technique for MIC determination, reported that isolates were either killed at concentrations of trimethoprim near the MIC or were not killed at any concentration tested. When trimethoprim was bactericidal, the combination of trimethoprim and sulphamethoxazole (TMP-SMZ) was also always bactericidal. They also noted a correla- 
tion between in-vitro bactericidal activity of sulphamethoxazole and its previously documented efficacy in eliminating nasopharyngeal carriage of some of the type-b isolates studied. Yogev and Moxon (1982) reported that type-b isolates which were not killed on exposure to TMP-SMZ elaborated significantly more type-b polysaccharide material than organisms sensitive to killing. After removal of the polysaccharide by physical methods, isolates were more easily killed by TMP-SMZ but reverted to their original phenotype after growth which allowed re-accumulation of capsular material.

Although both co-trimoxazole and trimethoprim alone are still used for non-invasive $H$. influenzae infections, particularly in patients hypersensitive to ampicillin, little is known about the mechanisms of resistance to these agents in this species. Since resistance to other antimicrobials documented in $H$. influenzae is frequently mediated by mechanisms which have much in common with those found in the enterobacteria, it has been widely presumed that the anti-folate agents are no exception. However, de Groot et al. (1988) have recently examined 10 non-capsulate trimethoprim-resistant isolates, none of which contained visually detectable extrachromosomal DNA. A DNA probe constructed from a subclone of the trimethoprim R-determinant of one isolate hybridised with the chromosomal DNA of both trimethoprim-sensitive and -resistant $H$. influenzae but not with Escherichia coli containing genes coding for dihydrofolate reductases (DHFRs) 1,2 and 3 or with trimethoprim-resistant strains of Neisseria. From an observation that DHFR activity was significantly higher in a trimethoprim-resistant strain than in an isogenic sensitive counterpart, it was suggested that resistance was associated with an overproduction of DHFR - a mechanism occasionally observed amongst enterobacteria (Steen and Skold, 1985). Further elucidation of these observations is awaited.

\section{Erythromycin}

Current prevalence of erythromycin resistance in the UK is unknown but with the NCCLS (National Committee for Clinical Laboratory Standards) breakpoint of $8 \mathrm{mg} / \mathrm{L}$, a recent study in the USA reported that $50 \%$ of isolates were resistant (Doern et al., 1988) compared with only 6\% of French isolates examined by Dabernat $e t$ al. in 1986. Machka et al. (1988) found a variation across Europe from $27 \%$ in the Netherlands to $1 \%$ in both Austria and the UK.

Fernandes et al. (1987) reported that, with
$0.5 \mathrm{mg} / \mathrm{L}$ and $8 \mathrm{mg} / \mathrm{L}$ as susceptible and resistant breakpoints, respectively, in-vitro MIC values correlated well with successful treatment of septicaemia in a mouse model but only when certain media were used and plates were incubated in air. It is clear that the extrapolation of in-vitro susceptibility to the likely clinical efficacy of erythromycin against $H$. influenzae is not straightforward and the usefulness of erythromycin is somewhat dubious.

\section{Rifampicin}

Opinion remains divided as to the role of rifampicin in prophylaxis against type-b infections among close contacts of an index case (Granoff $e t$ al., 1979; Eskola et al., 1987). Nicolle et al. (1982) documented a high prevalence of rifampicinresistant non-capsulate $H$. influenzae isolated from subjects after prophylaxis and suggested that their failure to find similar type-b isolates was due solely to the overall low prevalence of these strains in their specimens. Doern et al. (1988) found that $1 \%$ of USA isolates were resistant to rifampicin and Campos and Garcia-Tornel (1987) identified only one such isolate among 83 isolates resistant to ampicillin and chloramphenicol. A high frequency of mutation in-vitro to rifampicin resistance was reported among type-b isolates by Mendelman et al. (1982) who suggested that the variable degree of resistance observed might indicate that more than one mechanism was involved.

\section{4-Quinolones}

The 4-quinolones will undoubtedly be used increasingly for both invasive and non-invasive $H$. influenzae infections and may be of particular use in areas where multi-resistance is a problem. Ciprofloxacin appears to be highly active in vitro and effective in respiratory infections where $H$. influenzae is the predominant organism in the sputum (Raoof et al., 1986). Resistance to 4quinolones is not a problem at present.

\section{Multi-resistant isolates}

Type-b and non-capsulate isolates of $H$. influenzae resistant to four or more antimicrobial agents have been reported from many countries but are generally uncommon $(0.6 \%$ of isolates in the UK survey in 1986). Where multiply-resistant type-b isolates are a local problem, they do not appear to constitute a single clone. The Spanish isolates studied by Campos et al. (1987) collected from index cases of 
meningitis and carriers in four day-care centres during 1985, varied in OMP and plasmid restriction-endonuclease profiles.

\section{Prospects}

Despite the existence of resistance problems, a considerable choice of antimicrobial agents is still available for treatment of the majority of invasive and non-invasive $H$. influenzae infections. Nevertheless, a vaccine which would effectively prevent invasive disease by type-b strains in the age group

\section{REFERENCES}

Barany F, Kahn M E 1985 Comparison of transformation mechanisms of Haemophilus parainfluenzae and Haemophilus influenzae. Journal of Bacteriology 161 : 72-79.

Bell S M, Plowman D 1980 Mechanisms of ampicillin resistance in Haemophilus influenzae from respiratory tract. Lancet 1 : 279-280.

Burns J L, Mendelman P M, Levy J, Stull T L, Smith A L 1985 A permeability barrier as a mechanism of chloramphenicol resistance in Haemophilus influenzae. Antimicrobial Agents and Chemotherapy 27: 46-54.

Campos J, Garcia-Tornel S, Gairi J M, Fabregues I 1986 Multiply resistant Haemophilus influenzae type b causing meningitis: comparative clinical and laboratory study. Journal of Paediatrics 108: 897-902.

Campos J, Garcia-Tornel S 1987 Comparative susceptibilities of ampicillin and chloramphenicol resistant Haemophilus influenzae to fifteen antibiotics. Journal of Antimicrobial Chemotherapy 19: 297-301.

Campos J, Garcia-Tornel S, Musser J M, Selander R K, Smith A L 1987 Molecular epidemiology of multiply resistant Haemophilus influenzae type b in day care centers. Journal of Infectious Diseases 156: 483-489.

Dabernat H, Delmas C, Lareng M B 1986 Prevalence de la resistance aux antibiotiques des Haemophilus influenzae isoles en France: un an d'activite du reseau de surveillance des infections a $H$. influenzae. Pathologie et Biologie 34: 372 378

Doern G V, Jorgensen J H, Thornsberry C, Preston D A 1986 Prevalence of antimicrobial resistance among clinical isolates of Haemophilus influenzae: a collective study. Diagnostic Microbiology and Infectious Disease 4: 95-107.

Doern G V et al. 1988 National collaborative study of the prevalence of antimicrobial resistance among clinical isolates of Haemophilus influenzae. Antimicrobial Agents and Chemotherapy 32: 180-185.

Eskola J et al. 1987 Secondary cases of invasive disease caused by spread of Haemophilus influenzae type b. Journal of Infection 14: 233-236.

Fernandes P B et al. 1987 Susceptibility testing of macrolide antibiotics against Haemophilus influenzae and correlation of in vitro results with in vivo efficacy in a mouse septicaemia model. Antimicrobial Agents and Chemotherapy 31: 12431250 .

de Graaff J, Elwell L P, Falkow S 1976 Molecular nature of two beta-lactamase-specifying plasmids isolated from Haemophilus influenzae type b. Journal of Bacteriology 126: 439446 . most at risk ( $<18$ months) would circumvent many therapeutic problems arising from antimicrobial resistance amongst type- $b$ isolates (Granoff and Munson, 1986). Meanwhile, the expanding range of orally administered compounds, will facilitate treatment of non-invasive infections caused by type-b and non-capsulate isolates of $H$. influenzae and some of the newer injectable compounds will probably prove to be of particular value for serious infections caused by isolates resistant to many of the previously available antimicrobial agents.

GranoffD M, Gilsdorf J, Gessert C, Basden M 1979 Haemophilus influenzae type b disease in a day care center: eradication of carrier state by rifampicin. Paediatrics 63: 397-401.

Granoff D M, Munson R S 1986 Prospects for prevention of Haemophilus influenzae type $\mathrm{b}$ disease by immunization. Journal of Infectious Diseases 153: 448-461.

de Groot R, Campos J, Moseley S L, Smith A L 1988 Molecular cloning and mechanism of trimethoprim resistance in Haemophilus influenzae. Antimicrobial Agents and Chemotherapy 30: 477-484

Gunn B A, Woodall J B, Jones J F, Thornsberry C 1974 Ampicillin-resistant Haemophilus influenzae. Lancet 2 : 845.

Howard A J, Hince C J, Williams J D 1978 Antibiotic resistance in Streptococcus pneumoniae and Haemophilus influenzae. British Medical Journal 1: 1657-1660.

Jahn G, Laufs R, Kaulfers P M, Kolenda H 1979 Molecular nature of two Haemophilus influenzae $\mathrm{R}$ factors containing resistances and the multiple integration of drug resistance transposons. Journal of Bacteriology 138: 584-597.

Kirven L A, Thornsberry C 1978 Minimum bactericidal concentration of sulphamethoxazole-trimethoprim for $\mathrm{Hae}$ mophilus influenzae: correlation with prophylaxis. Antimicrobial Agents and Chemotherapy 14: 731-736.

van Klingeren B, van Embden J D A, Dessens-Kroon M 1977 Plasmid-mediated chloramphenicol resistance in Haemophilus influenzae. Antimicrobial Agents and Chemotherapy 11 : 383-387.

Levy S B, Buu-Hoi A, Marshall B 1984 Transposon Tn10-like tetracycline resistance determinants in Haemophilus parainfluenzae. Journal of Bacteriology 160: 87-94.

Loeb M R, Smith D H 1980 Outer membrane protein composition in disease isolates of Haemophilus influenzae: pathogenic and epidemiological implications. Infection and Immunity 30: 709-717.

Machka K et al. 1988 Distribution and resistance patterns of Haemophilus influenzae: a European cooperative study. European Journal of Clinical Microbiology 7: 14-24.

Makover D S, Wright R, Telep E 1981 Penicillin-binding proteins in Haemophilus influenzae. Antimicrobial Agents and Chemotherapy 19: 584-588.

Markowitz S M 1980 Isolation of an ampicillin-resistant, nonbeta-lactamase producing strain of Haemophilus influenzae. Antimicrobial Agents and Chemotherapy 17: 80-83.

Marshall B, Roberts M, Smith A, Levy S B 1984 Homogeneity of transferable tetracycline-resistance determinants in Haemophilus species. Journal of Infectious Diseases 149: 1028-1029.

Medeiros A A, O'Brien T F 1975 Ampicillin-resistant Haemophilus influenzae type b possessing a TEM-type $\beta$-lactamase 
but little permeability barrier to ampicillin. Lancet 1 : 716 719.

Medeiros A A, Levesque R, Jacoby G A 1986 An animal source for the ROB-1 $\beta$-lactamase of Haemophilus influenzae type b. Antimicrobial Agents and Chemotherapy 29: 212-215.

Mendelman P M, Roberts M C, Smith A L 1982 Mutation frequency of Haemophilus influenzae to rifampicin resistance. Antimicrobial Agents and Chemotherapy 22: 531-533.

Mendelman P M, Doroshow C A, Gandy S L, Syriopoulou V, Weigen C P, Smith A L 1984a Plasmid-mediated resistance in multiply resistant Haemophilus influenzae type b causing meningitis: molecular characterization of one strain and review of the literature. Journal of Infectious Diseases 150: 30-39.

Mendelman P M, Chaffin D O, Stull T L, Rubens C E, Mack K $\mathrm{D}$, Smith A L $1984 b$ Characterization of non- $\beta$-lactamasemediated ampicillin resistance in Haemophilus influenzae. Antimicrobial Agents and Chemotherapy $26: 235-244$.

Mendelman P M, Syriopoulou V P, Gandy S L, Ward J I, Smith A L 1985 Molecular epidemiology of plasmid-mediated ampicillin-resistance in Haemophilus influenzae type b isolates from Alaska. Journal of Infectious Diseases 151: 1061-1072.

Mendelman P M, Chaffin D O, Musser J M, de Groot R, Serfass D A, Selander R K 1987 Genetic and phenotypic diversity among ampicillin-resistant, non- $\beta$-lactamase-producing, nontypeable Haemophilus influenzae isolates. Infection and Immunity 55: 2585-2589.

Murphey-Corb M, Nolan-Willard M, Daum R S 1984 Integration of plasmid DNA coding for beta-lactamase production in the Haemophilus influenzae chromosome. Journal of Bacteriology 160 : 815-817.

Nicolle L E et al. 1982 Emergence of rifampin-resistant Haemophilus influenzae. Antimicrobial Agents and Chemotherapy 21 : 498-500.

Offit P A, Campos J M, Plotkin S A 1982 Ampicillin-resistant, beta-lactamase-negative Haemophilus influenzae type b. Paediatrics 69: 230-232.

Parr T R, Bryan L E 1984 Mechanism of resistance of an ampicillin-resistant, $\beta$-lactamase-negative clinical isolate of Haemophilus influenzae type $\mathrm{b}$ to $\beta$-lactam antibiotics. Antimicrobial Agents and Chemotherapy 25 : 747-753.

Powell M, Williams J D $1987 a$ In vitro susceptibility of Haemophilus influenzae to cefixime. Antimicrobial Agents and Chemotherapy 31 : 1841-1842.

Powell M, Williams J D $1987 b$ In vitro activities of aztreonam, imipenem and amoxycillin-clavulanate against ampicillinresistant Haemophilus influenzae. Antimicrobial Agents and Chemotherapy 31: 1871-1873.

Powell M, Koutsia-Carouzou C, Voutsinas D, Seymour A, Williams J D 1987 Resistance of clinical isolates of
Haemophilus influenzae in United Kingdom 1986. British Medical Journal 295: 176-179.

Powell M, Livermore D M 1988 Mechanisms of chloramphenicol resistance in Haemophilus influenzae in the United Kingdom. Journal of Medical Microbiology 27 : 89-93.

Powell M, Williams J D 1988 Detection of ampicillin-resistant Haemophilus influenzae in United Kingdom laboratories. Journal of Clinical Pathology 41: 716-719.

Raoof S, Wollschlager C, Khan F 1986 Treatment of respiratory tract infections with ciprofloxacin. Journal of Antimicrobial Chemotherapy 18 (SuppI D): 139-145.

Reid A J, Simpson I N, Harper P B, Amyes S G B 1987 Ampicillin resistance in Haemophilus influenzae: identification of resistance mechanisms. Journal of Antimicrobial Chemotherapy 20: 645-656.

Roberts M C, Smith A L 1980 Molecular characterization of 'plasmid-free' antibiotic resistant Haemophilus influenzae. Journal of Bacteriology 144: 476-479.

Roberts M C, Swenson C D, Owens L M, Smith A L 1980 Characterization of chloramphenicol-resistant Haemophilus influenzae. Antimicrobial Agents and Chemotherapy 18: 610615.

Roberts M, Corney A, Shaw W V 1982 Molecular characterization of three chloramphenicol acetyltransferases isolated from Haemophilus influenzae. Journal of Bacteriology 151 : 737-741.

Rubin L G, Medeiros A A, Yolken R H, Moxon E R 1981 Ampicillin treatment failure of apparently $\beta$-lactamasenegative Haemophilus influenzae type b meningitis due to novel $\beta$-lactamase. Lancet 2: $1008-1010$.

Serfass D A, Mendelman P M, Chaffin D O, Needham C A 1986 Ampicillin resistance and penicillin-binding proteins of Haemophilus influenzae. Journal of General Microbiology 132: 2855-2861.

Steen R, Skold O 1985 Plasmid-borne or chromosomallymediated resistance by $\operatorname{Tn} 7$ is the most common response to ubiquitous use of trimethoprim. Antimicrobial Agents and Chemotherapy 27: 933-937.

Stuy J H 1980 Chromosomally integrated conjugative plasmids are common in antibiotic resistant Haemophilus influenzae. Journal of Bacteriology 142: 925-930.

Vachon V, Lyew D J, Coulton J W 1985 Transmembrane permeability channels across the outer membrane of Haemophilus influenzae type b. Journal of Bacteriology 162 : 918-924.

Yogev R, Moxon E R 1982 Elaboration of type b capsule by Haemophilus influenzae as a determinant of pathogenicity and impaired killing by trimethoprim-sulphamethoxazole. Journal of Clinical Investigation 69: 658-665. 